\title{
PREVALENCIA DE GESTACIÓN CON RECIÉN NACIDO VIVO EN ADOLESCENTES DE TUNJA, COLOMBIA, 2011-2015
}

\section{Prevalence of pregnancies resullting in live births in adolescents in Tunja, Colombia, $2011-2015$ \\ Juan Manuel Ospina-Díaz, MD, $M_{S c^{1}}$; Lina Fernanda Barrera-Sánchez, $M_{S c^{2}}$; Cindy Lorena López-Ortega, $M D^{3}$}

Recibido: febrero 8/16 - Aceptado: noviembre 16/16

\section{RESUMEN}

Objetivo: estimar la frecuencia y las características del embarazo y el parto, con producto final vivo, en adolescentes; además, identificar algunos factores relacionados con el embarazo con recién nacido vivo en adolescentes en la ciudad de Tunja durante el periodo 2011-2015.

Materiales y métodos: estudio poblacional, descriptivo, de corte transversal. Se incluyeron los registros de nacimientos ocurridos en menores de 19 años en Tunja, durante enero de 2011 y octubre de 2015, a partir de la base de datos de la Secretaría de Protección Social municipal. Se evaluaron variables sociodemográficas de los padres, la atención durante la gestación y las características del recién nacido. Se estimaron las frecuencias absolutas y relativas de variables discretas. La información de las variables continuas se resume mediante medidas de

1 Médico; magíster en Epidemiología. Profesor Titular, Escuela de Medicina, Universidad Pedagógica y Tecnológica de Colombia, Tunja, Colombia. Investigador, Grupo de Investigación en Salud Pública (GISP), Tunja (Colombia). juan.ospina@uptc.edu.co

2 Enfermera; magíster en Salud Pública. Profesora Asistente, Escuela de Enfermería, Universidad Pedagógica y Tecnológica de Colombia, Tunja, Colombia. Investigador, Grupo de Investigación en Salud Pública (GISP), Tunja (Colombia).

3 Médica interna, Escuela de Medicina, Universidad Pedagógica y Tecnológica de Colombia, Tunja, Boyacá. Investigadora, Grupo de Investigación en Salud Pública (GISP), Tunja (Colombia). tendencia central y dispersión. Se calculó la razón de gestantes con recién nacido vivo en mujeres en edad fértil y en adolescentes

Resultados: se presentó un total de 1518 partos en una población de 13.408 adolescentes (a mitad de periodo), lo que representa una prevalencia global de 11,32\%, que varió entre 10,6\% en el 2105 y $12 \%$ en el 2011. Como factores asociados con el parto adolescente se encontró mayor proporción de afiliación al SGSSS por régimen subsidiado, bajo nivel de escolaridad de la gestante y la pareja, hábitat rural, carencia de pareja estable.

Conclusiones: la prevalencia de embarazo adolescente llevado a término en Tunja es inferior a la media nacional reportada. Es recomendable profundizar en el análisis de los determinantes de la frecuencia de embarazo y parto en adolescentes, que podrían contribuir a mejorar las estrategias de intervención en otras regiones de mayor prevalencia. Palabras clave: embarazo en adolescentes, prevalencia, obstetricia.

\section{ABSTRACT}

Objective: To estimate the frequency and characteristics of pregnancy and delivery with a live birth product in adolescents, and to identify some factors associated with pregnancy and the final 
birth outcome in adolescentes in Tunja between 2011 and 2015.

Materials and methods: Cross-sectional, descriptive population study. The study included the records of live births in girls under 19 years of age in Tunja between January 2011 and October 2015 taken from the database of the Municipal Social Protection Office. The variables included social and demographic characteristics of the parents, care during pregnancy, and characteristics of the newborn. Absolute and relative frequencies of discrete variables were estimated together with the corresponding central trend and scatter valuations. The ratio of pregnant women with a live birth to women in childbearing age and adolescents was estimated.

Results: Overall, 1518 deliveries were recorded in a population of 13408 adolescents (half way into the period), accounting for an overall prevalence of $11.32 \%$, ranging between $10.6 \%$ in 2105 and $12 \%$ in 2011. The factors found to be associated with childbirth in adolescents was a higher proportion of affiliation to the Social Security System in Health (SGSSS) through the subsidised regime, low level of schooling of the pregnant girl and the partner, living in the rural area, and the lack of a stable partner.

Conclusions: The prevalence of term teenage pregnancy in Tunja is lower than the reported national average. It is advisable to conduct a more in-depth analysis of the determinants of pregnancy and childbirth frequency in adolescents, in order to try to improve intervention strategies in other regions with a higher prevalence.

Key words: Teenage pregnancy, prevalence, obstetrics.

\section{INTRODUCCIÓN}

Acorde con la legislación vigente en Colombia, la adolescencia se entiende como el periodo de la vida comprendido entre los 12 y los 18 años de edad (1). En la normativa se impone al sector salud la obligación de desarrollar programas para la prevención del embarazo no deseado en este grupo de edad. El Instituto Colombiano de Bienestar Familiar (ICBF) reporta que en Colombia, $13 \%$ de las adolescentes menores de 15 años ya ha iniciado vida sexual activa, 19,5\% del grupo entre 15 y 19 años ya ha tenido al menos un embarazo, $16 \%$ ya son madres y $4 \%$ está esperando su primer hijo. Como factor asociado se reporta que en 99,2\% de los casos el papá es un adulto y solo en $0,8 \%$ es también adolescente (2).

Se ha informado que para el año 2008, el $11 \%$ de todos los embarazos ocurridos en el mundo se presentaron en jóvenes entre 15 y 19 años de edad; es importante resaltar que el $95 \%$ de todos estos embarazos ocurrieron en países de bajos y medios ingresos (3). Respecto a la distribución geográfica, las frecuencias oscilaban entre 5 por 1000 en Asia oriental y 121 por 1000 en el África subsahariana, durante el año 2007. No obstante, la tasa de natalidad entre adolescentes se ha reducido desde 60 por 1000 en 1990 hasta 48 por 1000 en 2007 (3). En contraste con esta disminución, el número absoluto de nacimientos en madres adolescentes ha disminuido menos debido al aumento de este grupo de edad. En algunos países, la proporción de nacimientos en adolescentes se ha incrementado, probablemente por la reducción de la fertilidad en las mujeres mayores (3).

En relación con el ámbito espacial, se encuentra que el embarazo adolescente es más frecuente en las áreas rurales (26,7\%) que en escenarios urbanos (17,3\%); también es un factor importante el nivel educativo, ya que más de la mitad de los casos ocurren en jovencitas sin ninguna educación o con menos de la primaria terminada (4). Se reconoce, asociado al embarazo y parto adolescente, la menarquia temprana, inicio precoz de relaciones sexuales, familia disfuncional, migración o desplazamiento reciente, estrato socioeconómico bajo, ausencia de educación sexual integral, limitado acceso a tecnologías anticonceptivas eficaces (5); además, un alto porcentaje de madres adolescentes han abandonado los estudios antes de embarazarse y han debido asumir roles de cuidado de los hermanos, en reemplazo de la mamá, lo que las lleva a ser simultáneamente "madres-hijas" $(4,6)$. En los países 
en vía de desarrollo coexisten aspectos psicológicos que influyen sobre las gestaciones y los partos en adolescentes, especialmente cuando es tendencia cultural el matrimonio a corta edad; también puede ocurrir que, en algunas áreas, las niñas quieran demostrar ser fértiles como una afirmación de su feminidad, incluso también para consolidar una relación afectiva a través del embarazo (7).

En el campo de la atención clínica del parto en la adolescente gestante se reconoce un riesgo incrementado de ciertas complicaciones como preeclampsia, eclampsia, ruptura prematura de membranas, endometritis puerperal y sangrado profuso en el posparto (8). Por otra parte, se reporta que el aborto espontáneo en adolescentes embarazadas puede oscilar entre el 12 y $28 \%$ de los $\operatorname{casos}(4,9,10)$.

Se ha demostrado que el embarazo y posterior parto en mujeres adolescentes es un factor que permite que se perpetúe el ciclo de la pobreza, disminuyendo la probabilidad de las jóvenes para alcanzar sus aspiraciones en los planos educativo, laboral y socioeconómico (8); la maternidad precoz contribuye significativamente a una mayor mortalidad materna y fetal (11); además, entre los niños producto de maternidad adolescente es mayor el riesgo de abusos físicos o sexuales, además de una mayor frecuencia de alteraciones del comportamiento (11).

Si bien se considera que el embarazo y el parto en jóvenes mujeres adolescentes constituye un problema de salud pública, es importante considerar que, en la actualidad, cerca de la mitad de la población mundial tiene menos de 25 años; además, una de cada 5 personas es un adolescente que está entre los 10 y los 19 años de edad (12). Los agentes de salud están obligados a considerar que en esta etapa de la vida ocurre un apreciable número de fenómenos en los planos físico, emocional, cognitivo, social y psicológico, que buscan que en las jóvenes se consoliden una serie de aptitudes y capacidades que deben permitir un tránsito sin contratiempos hacia la madurez. Por esta razón, este grupo de edad en particular es de alto riesgo en términos de salud sexual y reproductiva, habida cuenta de la existencia de condiciones subjetivas que pueden llevar a la toma de decisiones apresuradas capaces de modificar la vida, generando pérdida de oportunidades y modificando las expectativas hacia el futuro (13).

Hay escasa información en el medio local sobre la frecuencia del embarazo en adolescentes, sus determinantes sociales, la magnitud del fenómeno de la interrupción voluntaria del embarazo en este grupo de edad y de las complicaciones maternas y perinatales en este grupo de gestantes. En consecuencia, este estudio tiene como objetivo describir la prevalencia del embarazo en adolescentes, sus características y el resultado del parto, en la ciudad de Tunja, Colombia.

\section{MATERIALES Y MÉTODOS}

Estudio poblacional descriptivo, de corte transversal. Se incluyeron mujeres en edad fértil, residentes en la ciudad de Tunja, que tuvieron un parto, entre enero de 2011 y octubre de 2015. Tunja es la capital del departamento de Boyacá, ubicado al nororiente de Bogotá, capital de Colombia. Del total de mujeres se determinaron aquellas entre 12 y 18 años que tuvieron un parto con producto final vivo; adicionalmente, del registro del Departamento Administrativo Nacional de Estadísticas (DANE), se obtuvo el total de óbitos fetales ocurridos durante el mismo periodo. Se hizo muestreo secuencial estricto.

Los registros de proyección poblacional del DANE para la ciudad de Tunja se obtuvieron de la página web de esta institución (14). La relación de nacimientos se obtuvo del registro institucional de la Secretaría de Protección Social de Tunja, mediante solicitud formal cursada a esta entidad. La información se almacenó en una base de datos en Excel.

Las variables generales incluidas fueron la localidad de residencia, el origen (urbano-rural); la edad y escolaridad y el estado civil de la madre; el número de consultas prenatales, partos y embarazos previos; el tipo de parto; el sexo, el peso y la talla 
del recién nacido; la edad del padre; la escolaridad y el último año aprobado de la madre. Adicionalmente, de la misma base de datos de la Secretaría de Protección Social de Tunja se obtuvo y analizó información referida a la edad del padre, así como su escolaridad. Además, el registro de atención prenatal y resultado final (tipo de parto, antropometría del recién nacido, vitalidad).

En las variables continuas se analizaron medidas de tendencia central y dispersión; en las variables discretas se analizaron los porcentajes por categoría. Con base en la población femenina estimada a mitad de periodo se calculó la correspondiente razón de gestantes con recién nacido vivo en el grupo general (recién nacidos vivos/mujeres en edad fértil) por años la razón de gestantes con recién nacido vivo en adolescentes (recién nacidos vivos en mujeres de 12 a 18 años/mujeres de 12 a 18 años) por año. La prevalencia de embarazo en gestantes se obtuvo del número total de partos en adolescentes/total de partos en todas las mujeres en edad fértil. Como información complementaria, y teniendo en cuenta el rango de edad establecido por la Organización Panamericana de la Salud (OPS) en su definición de adolescencia, y con el propósito de establecer comparaciones con los reportes de otros países y de la Encuesta Nacional de Salud (ENSAD), se estimó la razón de prevalencia de gestantes con recién nacidos vivos en la población en edad fértil y en la población de gestantes de 15 a 19 años de edad.

De manera exploratoria se buscó identificar algunos factores clínicos y socioeconómicos relacionados con el embarazo y parto en adolescentes mediante las pruebas $t$ de Student en variables continuas, y chi cuadrado en el caso de las variables discretas.

Aspectos éticos. El estudio fue aprobado por el Comité de Ética institucional; se preservaron los principios de confidencialidad y privacidad para todos los sujetos incluidos.

\section{RESULTADOS}

Acorde con las proyecciones del DANE, la población de mujeres en edad fértil en Tunja a mitad de periodo estudiado ascendió a 53.572; de ellas 11.888 (22,1\%) eran adolescentes. En el periodo comprendido entre enero de 2011 y octubre de 2015 ocurrieron un total de 12.846 partos, de ellos 1626 en los que la edad de la madre era igual o menor a 18 años. La prevalencia de parto con feto vivo en adolescentes por año varió entre el 10,6 y el 12,4\% (tabla 1). La prevalencia de

\begin{tabular}{|c|c|c|c|c|c|c|c|}
\hline Año & $\begin{array}{c}\text { Mujeres } \\
\text { en edad } \\
\text { fértil }\end{array}$ & $\begin{array}{l}\text { Recién } \\
\text { nacidos } \\
\text { vivos }\end{array}$ & Razón & $\begin{array}{c}\text { Mujeres } \\
\text { entre } \\
\text { 12-18 años }\end{array}$ & $\begin{array}{c}\text { Recién } \\
\text { nacidos vivos } \\
\text { Adolescentes } \\
\text { 12-18 años }\end{array}$ & Razón & $\begin{array}{c}\text { Prevalencia } \\
\text { partos en } \\
\text { Adolescentes }\end{array}$ \\
\hline 2011 & 52.575 & 2601 & 4,94 & 11.351 & 324 & 2,85 & 12,4 \\
\hline 2012 & 53.172 & 2615 & 4,91 & 11.613 & 309 & 2,66 & 11,8 \\
\hline 2013 & 53.772 & 2646 & 4,92 & 11.888 & 328 & 2,76 & 12,4 \\
\hline 2014 & 54.350 & 2693 & 4,95 & 12.131 & 315 & 2,60 & 11,7 \\
\hline 2015 & 54.896 & 2291 & $4,17^{*}$ & 12.325 & 242 & $1,96^{*}$ & 10,6 \\
\hline Total & & 12.846 & & & 1518 & & 11,8 \\
\hline
\end{tabular}

* Datos sin proyección estimados hasta octubre 31 de 2015.

Fuentes: DANE, Proyecciones de población por edades simples, Tunja 2011-2015; Base de Datos Secretaría de Salud de Tunja. 
gestantes entre 15 y 19 años varía ligeramente con valores entre 10,2 y 12,01\% (tabla 2). El porcentaje anual de mortalidad fetal osciló entre 2,15 y 6,62 \%, con una estimación global de 4,05 \% para el mismo grupo de edad (tabla 3).

La media de edad de la población estudiada fue de 26,25 años ( $\mathrm{SD}=6,5)$, mientras que el promedio del grupo de adolescentes fue 16,9 años
$(\mathrm{SD}=1,11)$ y de las mujeres mayores de 18 años, 27,5 años $(\mathrm{SD}=5,87)$; el 11,6\% $(\mathrm{n}=156)$ de las adolescentes que tuvieron un parto tenían 15 años o menos. Respecto de la escolaridad de las madres adolescentes, 12,97\% $(\mathrm{n}=197)$ había estudiado hasta quinto de primaria; 42,7\% $(n=649)$ primaria y hasta noveno grado; y 31,7\% $(n=480)$ había terminado la secundaria.

Tabla 2.

Razón de prevalencia de gestantes con recién nacido vivo en Tunja (Colombia), 2011-2015, en mujeres fértiles y en adolescentes de 15 a 19 años, según el rango de edad definido por la OPS para adolescencia

\begin{tabular}{|l|c|c|c|c|c|c|c|}
\hline Año & $\begin{array}{c}\text { Mujeres } \\
\text { en edad } \\
\text { fértil }\end{array}$ & $\begin{array}{c}\text { Recién } \\
\text { nacidos } \\
\text { vivos }\end{array}$ & Razón & $\begin{array}{c}\text { Mujeres } \\
\text { entre } \\
15-19 \text { años }\end{array}$ & $\begin{array}{c}\text { Recién nacidos vivos } \\
\text { Adolescentes } \\
15-19 \text { años }\end{array}$ & Razón & $\begin{array}{c}\text { Partos en } \\
\text { Adolescentes } \\
\text { (\%) }\end{array}$ \\
\hline 2011 & 52.575 & 2601 & 4,94 & 9.349 & 311 & 3,33 & 11,95 \\
\hline 2012 & 53.172 & 2615 & 4,91 & 9.609 & 293 & 3,05 & 11,20 \\
\hline 2013 & 53.772 & 2646 & 4,92 & 8.177 & 318 & 3,89 & 12,01 \\
\hline 2014 & 54.350 & 2693 & 4,95 & 8.431 & 312 & 3,70 & 11,6 \\
\hline 2015 & 54.896 & 2291 & $4,17 *$ & 8.653 & 235 & $2,71 *$ & 10,2 \\
\hline Total & & 12.846 & & & 1469 & & 11,4 \\
\hline
\end{tabular}

* Datos sin proyección estimados hasta octubre 31 de 2015.

Fuentes: DANE, Proyecciones de población por edades simples Tunja 2011-2015; Base de Datos Secretaría de Salud de Tunja.

Tabla 3.

Proporción de mortalidad fetal, 2011-2015, en mujeres fértiles y en adolescentes de 15-19 años, según rango de edad definido por la OPS para adolescencia

\begin{tabular}{|c|c|c|c|c|c|c|}
\hline Año & $\begin{array}{c}\text { Total } \\
\text { nacimientos } \\
\text { Tunja }\end{array}$ & $\begin{array}{c}\text { Total } \\
\text { defunciones } \\
\text { fetales }\end{array}$ & $\begin{array}{c}\text { Tasa } \\
\text { defunciones } \\
\text { fetales Tunja }\end{array}$ & $\begin{array}{c}\text { Total, } \\
\text { nacimientos } \\
15-19 \text { años }\end{array}$ & $\begin{array}{c}\text { Total, } \\
\text { defunciones } \\
\text { fetales 15-19 } \\
\text { años }\end{array}$ & $\begin{array}{c}\text { Tefunciones } \\
\text { fetales } 15-19 \\
\text { años }\end{array}$ \\
\hline 2011 & 2663 & 88 & 3,3 & 325 & 14 & 4,30 \\
\hline 2012 & 2684 & 139 & 5,17 & 302 & 9 & 2,98 \\
\hline 2013 & 2697 & 125 & 4,63 & 325 & 7 & 2,15 \\
\hline 2014 & 2733 & 88 & 3,22 & 326 & 14 & 4,30 \\
\hline 2015 & 2771 & 85 & 3,06 & 302 & 20 & 6,62 \\
\hline Total & 13548 & 525 & 3,87 & 1580 & 64 & 4,05 \\
\hline
\end{tabular}

Fuentes: DANE, Estadísticas vitales nacimientos y defunciones; Tunja 2011-2015.

Disponible en: http://www.dane.gov.co/index.php/estadisticas-por-tema/demografia-y-poblacion/nacimientos-y-defunciones. Base de Datos Secretaría de Salud de Tunja. 
En lo que tiene que ver con la estructura del ámbito de familia, 693 gestantes adolescentes (45,6\%) no tenían una pareja estable, y 272 (17,9\%) estaban casadas o llevaban más de dos años conviviendo con el padre del recién nacido. Un total de 553 (36,4\%) no estaban casadas o llevaban menos de dos años conviviendo con su pareja.

La mediana de consultas prenatales fue de 6 (rango 0-17); 260 (17,1\%) de ellas tuvieron entre 0 y 3 controles, y 209 (13,8\%) asistieron a 9 o más controles. La media de edad gestacional fue 38,4 semanas (mediana: 39; rango 25-42); el peso promedio de los recién nacidos fue 2962,6 g $(\mathrm{SD}=456,9)$. De los recién nacidos, $172(11,3 \%)$ tuvieron un peso menor de $2500 \mathrm{~g}$, mientras que $123(8,1 \%)$ pesó más de 3500 g; la talla promedio fue 49,7 cm (SD = 2,8;); 11,1\%.

El 31,9\% (n = 484) de los partos fue por cesárea o instrumentados; 11,3\% ( $\mathrm{n}=171)$ de las adolescentes gestantes había tenido al menos un embarazo previo al presente; $10,01 \%(\mathrm{n}=152)$ cursaba su segundo embarazo; $1,05 \%(n=16)$ el tercero, mientras que $0,20 \%(n=3)$ el cuarto y más.

$\mathrm{Al}$ primer minuto, un puntaje APGAR igual o menor que 6 ocurrió en 32 gestantes adolecentes $(2,1 \%)$, y solo se mantuvo bajo a los 5 minutos en 6 recién nacidos $(0,4 \%)$.
La media de edad del padre del recién nacido fue 21,6 años ( $\mathrm{SD}=5,26)$; en 111 gestantes adolescentes (7,3\%) el compañero tenía más de 30 años de edad (rango 30-71 años). En 401 gestantes adolescentes (26,6\%) el padre tenía 18 años o menos de edad. En 3,4\% de los casos $(n=51)$ la madre adolescente era entre 1 y 3 años mayor que el padre. Entre los padres, 25,7\% (n=391) carecía de educación o tenía máximo cinco años de escolaridad; 29,1\% (n= 442) había cursado máximo hasta nueve años; 40,6 $(\mathrm{n}=586)$ había terminado el bachillerato y 6,25\% $(\mathrm{n}=92)$ había adelantado estudios superiores.

En cuanto a los factores socioeconómicos y clínicos asociados al embarazo en adolescente se encontraron la baja educación de los padres, pertenecer al régimen subsidiado por el Estado, vivir en área rural y no tener pareja estable (tabla 4).

\section{DISCUSIÓN}

La prevalencia de embarazo en adolescentes de 12 a 18 años en la ciudad de Tunja para el periodo 2011 a 2015 varió entre el 10,6 y el 12,4\%. Cuando se toman solo las adolescentes de 15 a 19 años, la frecuencia varía ligeramente entre el 10,2 y el 12,01\%. También se encuentra que la tasa de mortalidad fetal es ligeramente mayor en el grupo de

\begin{tabular}{|c|c|c|c|}
\hline \multirow[b]{2}{*}{ Variable } & \multicolumn{2}{|c|}{ Grupo } & \multirow[b]{2}{*}{ P } \\
\hline & $\begin{array}{l}\text { Adolescente (\%) } \\
\qquad(\mathrm{n}=1518)\end{array}$ & $\begin{array}{l}\text { Adulta (\%) } \\
(n=11328)\end{array}$ & \\
\hline Madre bachillerato incompleto & $484(31,8)$ & $4819(42,5)$ & $<0,0001$ \\
\hline Padre bachillerato incompleto & $713(46,9)$ & $3536(30,94$ & $<0,0001$ \\
\hline Régimen subsidiado & $944(62,2)$ & $4251(37,5)$ & $<0,0001$ \\
\hline Residencia rural & $134(8,8)$ & $557(4,9)$ & $<0,0001$ \\
\hline No tiene pareja estable & $693(45,6)$ & $1855(16,4)$ & $<0,0001$ \\
\hline
\end{tabular}


adolescentes cuando se compara con las gestantes mayores de 18 años, tal como se registra en la tabla 3; esta diferencia y su significado de riesgo para las adolescentes se ha documentado mediante revisión sistemática (15).

Por otra parte, se encontró que el embarazo en adolescentes está asociado en Tunja a la baja escolaridad tanto de la mujer como de su compañero, estar afiliada al régimen subsidiado por el Estado y no tener pareja estable. Respecto a la dinámica de atención de las adolescentes gestantes, se encuentra un bajo número de atenciones prenatales en el $17 \%$ de estas.

Los resultados de tasas anuales de embarazo y parto adolescente registradas en Tunja durante el periodo estudiado contrastan significativamente con los reportes consignados de Demografía y Salud en Colombia, así como con las estadísticas a nivel nacional en diferentes regiones, por ejemplo, los departamentos con mayores tasas son Amazonas (35,4\%), Putumayo (32\%), Vichada (31,3\%), La Guajira (25,8\%), Chocó (29,4\%), Nariño (21,8\%), Cesar $(25,8 \%)$ y Cauca $(23,8 \%)$ (16). Se estima que, a nivel mundial, entre 15 y $20 \%$ de todos los nacimientos ocurren en madres adolescentes, siendo esta participación mayor en los países en vía de desarrollo; en Perú, por ejemplo, la tasa de embarazo y parto en adolescentes asciende al 13,5 \% (17); en Brasil, en el periodo 2000-2006, la tasa de nacimientos en madres adolescentes fue de 20,6\%, similar a la tasa de Tanzania en el periodo 1999-2005, que fue de 18,0\% $(15,18)$. En el otro extremo de la balanza, Estados Unidos registró en 2013 un 2,65\%, cifra bastante baja, pero que no obstante es $50 \%$ mayor que la de Gran Bretaña y más del doble de la de Canadá (19).

En cuanto a los factores asociados al embarazo adolescente que se pudieron estimar en el presente estudio, encontramos que todos ellos concuerdan, en términos generales, con lo descrito en la indagación dada a conocer por Profamilia en 2013, en la que destacan como factores asociados el bajo nivel educativo y la accesibilidad a información confiable sobre métodos anticonceptivos, en contraste con el nivel socioeconómico de la familia, que entre 20052010 muestra cierta tendencia a la disminución. La carencia de una pareja estable también contribuye significativamente al riesgo de una gestación precoz entre las adolescentes (20). Resultados similares se reportan en un metaanálisis de casos y controles adelantado por investigadores de Brasil, en el que se destaca el bajo tiempo de permanencia en las aulas como determinante (15).

Respecto al nivel educativo, en el año 2014 este factor se reportó, en un estudio adelantado previamente en Boyacá, asociado a la ocurrencia del embarazo adolescente; ahí se destacan como factores educacionales ligados al fenómeno del embarazo adolescente, la deserción escolar temprana y las consecuentes dificultades para estructurar proyectos de vida a largo plazo (21). Esta apreciación es concordante con la necesidad de estructurar en los currículos escolares elementos que les brinden a las jóvenes el conocimiento, las competencias y los valores que les permitan asumir responsabilidad sobre su vida sexual y social (22).

En lo que tiene que ver con el papel que juegan la pobreza y la marginalidad, representadas por el aseguramiento subsidiado y la vivienda en el área rural, recién se han formulado hipótesis estructuradas bajo el criterio de la “teoría de la desesperación”, según la cual, la percepción de ausencia de oportunidades para la realización personal y económica lleva a las adolescentes a anticipar la decisión de la maternidad; esta teoría se postula en el mismo sentido para los adolescentes varones (23). Este aspecto está ligado no solo al bajo nivel educativo, sino también a las limitadas alternativas de desarrollo personal y social para las niñas; modelos educativos de baja calidad, particularmente en lo que tiene que ver con la salud sexual y reproductiva, que se reflejan en el no uso o uso intermitente de tecnología anticonceptiva; limitadas posibilidades de inserción laboral y ausencia de proyecto de vida, y metas personales definidas a mediano y largo plazo (24).

Una circunstancia que oscurece significativamente el panorama de la adolescente embarazada 
se relaciona con la ausencia de una pareja estable. Parece ser que la ausencia de apoyo afectivo y económico por parte del padre del neonato influye de manera significativa sobre el bienestar y la predisposición psicológica de la madre para afrontar el desafío de la maternidad, además de que cierra para ella el acceso a beneficios de tipo económico, legal y asistencial (25).

Más de la mitad asistió a un alto número de consultas prenatales, circunstancia que sugiere una infraestructura sanitaria adecuada, así como también preocupación por un desarrollo adecuado de las gestaciones con apoyo familiar a las adolescentes embarazadas, este hallazgo también contrasta con lo reportado en estudios similares, incluidos aquellos en los que se enfatiza en la importancia del abordaje integral del embarazo adolescente involucrando a la familia, $(26,27)$. La importancia del control prenatal ha puesto en evidencia la ventaja de reconocer oportunamente patologías de riesgo como infecciones, hipertensión y retardo del crecimiento intrauterino, las detectadas con más frecuencia en las adolescentes (25).

Como limitaciones de este estudio hay que considerar la carencia de información sobre las comorbilidades maternas y fetales, así como sobre la mortalidad perinatal, lo cual impide elaborar un perfil epidemiológico más profundo. No obstante, ya que se incluye la totalidad de partos registrados institucionalmente durante el periodo estudiado, se puede considerar que la inferencia sobre los hallazgos es estadísticamente confiable y pone en la palestra inquietudes que invitan a adelantar, en el futuro, análisis más detallados de los determinantes sociales, culturales y económicos que influyen sobre las tasas locales de embarazo adolescente.

\section{CONCLUSIONES}

Se encuentra una prevalencia de embarazo adolescente significativamente menor a la reportada en la media nacional. Como principales características relacionadas se encuentra la baja escolaridad de las adolescentes y de sus parejas, hábitat rural, carencia de pareja sexual estable y afiliación de tipo subsidiado o vinculado al SGSSS. Estos hallazgos sugieren la existencia de factores culturales no identificados que influyen sobre la menor frecuencia de embarazo adolescente en la ciudad de Tunja, cuando se la compara con otras ciudades del país. Se recomienda diseñar y desarrollar estudios analíticos que permitan identificar estos factores para mejorar las estrategias de intervención en la prevención del embarazo adolescente.

\section{AGRADECIMIENTOS}

Los autores hacen expreso reconocimiento y manifestación de gratitud a la Secretaría de Salud de Tunja, en particular a la doctora Gina Natale por su desinteresada colaboración y apoyo en el desarrollo de este estudio.

\section{REFERENCIAS}

1. República de Colombia, Ley 1098 de 2006.

2. República de Colombia. Ministerio de Salud y Protección Social. Embarazo adolescente en Colombia; 2013. [visitado 2016 Ene 01]. Disponible en: https://www. minsalud.gov.co/salud/Documents/embarazoadolescente/anexo-cifras-embarazo-adolescente-encolombiadocumentoICFB-jul-2013.pdf.

3. World Health Organization (WHO). Early marriages, adolescents and young pregnancies. Sixty-Fifth World Health Assembly provisional agenda item 13.4: New York. 2012 [visitado 2016 Ene 02]. Disponible en: http://apps.who.int/iris/handle/10665/23744

4. Valencia JG, Parra JM, Rey E, Escalante BY, Delgado MS. Causas del embarazo adolescente en los municipios de Acacías, Cúcuta, Pasto y Yopal durante el primer semestre del año 2014. Universidad Abierta y a Distancia (UNAD). Trabajo final curso de Fundamentos de Salud Pública. Tecnología de Regencia en Farmacia. Bogotá DC, 2014 [visitado 2016 Ene 03]. Disponible en: http://repository.unad. edu.co/ bitstream/10596/2627/1/1120366912.pdf

5. Díaz L, Espitia J, Prasca M, Puerta Y, Quintana C, Hernández ML. Prevalencia de embarazo en adolescentes escolarizados y sus factores predisponentes: Cartagena 
2012-2013. Tesis de Grado. Facultad de Enfermería Universidad de Cartagena. 2013 [visitado 2016 Nov 11]. Disponible en: http://190.242.62.234:8080/ jspui/handle/11227/2802

6. Villalobos-Hernández A, Campero L, Suárez-López L, Atienzo EE, Estrada F, De la Vara-Salazar E. Embarazo adolescente y rezago educativo: análisis de una encuesta nacional en México. Salud Pública Mex. 2015;57:135-43.

7. Hindin A, Rodríguez MI, Gonsalvez L, Say L. Adolescent health experience after abortion or deliver y (AHEAD trial): formative protocol for intervention development to prevent rapid, repeat pregnancy. Reproductive health. 2015 [visitado 2016 Ene 03]; 12:111. Disponible en: http://www.ncbi.nlm. nih.gov/ pmc/articles/PMC4666050/ Doi: 10.1186/ s12978015-0098-4.

8. Díaz LM, Chávez BM. Complicaciones maternas en adolescentes y adultas afiliadas al régimen subsidiado, 2012. Rev Fac Nac Salud Pública. 2015;33:206-17. DOI: 10.17533/udea.rfnsp.v33n2a08.

9. Corres M, Castro EV, Ortiz ME, Shaw RJ, Colin A, Ponce ER. Morbilidad materno fetal en adolescentes: experiencia en un hospital suburbano de México. An Med (Mex). 2013;58:175-9.

10. Okomura JA, Maticorena DA, Tejeda JE, MaytaTristan P. Embarazo adolescente como factor de riesgo para complicaciones obstétricas y perinatales en un hospital de Lima, Perú. Rev Bras Saúde Matern Infant. 2014;14:383-92.

11. Gómez-Mercado CA, Montoya-Vélez LP. Factores sociales, demográficos, familiares y económicos relacionados con el embarazo en adolescentes, área urbana, Briceño, 2012. Rev Salud Pública. 2014;16:394-406.

12. UNFPA. State of the world population 2003, making a billion counts: adolescents' health and right. New York: UNFPA; 2003.

13. Ahorlu CK, Pfeiffer C, Obrist B. Socio-cultural and economic factors influencing adolescents' resilience against the threat of teenage pregnancy: a crosssectional survey in Accra, Ghana. Reprod health. 2015;12:217. Doi: 10.1186/s12978-015-0113-9.
14. República de Colombia. Departamento Nacional de Planeación. Estimación y proyección de población nacional, departamental y municipal total por área 19852020 [visitado 2016 nov 28]. Disponible en: https:// www.dane.gov.co/index.php/estadisticas-por-tema/ demografia-y-poblacion/proyecciones-de-poblacion

15. Azevedo WF, Diniz MB, Fonseca ES, Azevedo LM, Evangelista CB. Complications in adolescent pregnancy: systematic review of the literature. Einstein (São Paulo). 2015;13:618-26.

16. República de Colombia. Ministerio de Salud y Protección Social. Embarazo adolescente en Colombia. 2013 [visitado 2016 Ene 03]. Disponible en: https:// www.minsalud.gov.co/salud/Documents/embarazoadolescente/anexo-cifras-embarazo-adolescente-encolombia-documentoICFB-jul-2013.pdf.

17. Okumura JA, Maticorena DA, Tejeda JE, Mayta P. Embarazo adolescente como factor de riesgo para complicaciones obstétricas y perinatales en un hospital de Lima, Perú. Rev Bras Saúde Matern Infant. 2014;14:383-92.

18. Muganyizi PS, Kidanto HL. Impact on change in maternal age composition on the incidence of caesarian section and low birth weight: analysis of delivery records at a tertiary hospital in Tanzania, 1999-2005. BMC Pregnancy and childbirth. 2009;9:30. Doi: 10.1186/1471-2393-9-30.

19. Manlove J, Fish H, Moore KA. Programs to improve adolescent sexual and reproductive health in the US: a review of the evidence. Adolesc Health Med Ther. 2015;6:47-79.

20. Flórez CE, Soto VE. Factores protectores y de riesgo del embarazo adolescente en Colombia. Bogotá: Profamilia; 2013 [visitado 2016 Ene 02]. Disponible en: http://www.profamilia.org.co/docs/ ESTUDIOS \%20A \%20PROFUNDIDAD \%20AF.pdf.

21. Ariza NE, Valderrama ML, Ospina JM. Caracterización del embarazo adolescente en dos ciudades de Boyacá, Colombia. Revista Virtual Universidad Católica del Norte. 2014 [visitado 2016 Nov 8]; 42:154-63. Disponible en: http://revistavirtual.ucn.edu.co/index. php/RevistaUCN/article/view/501/1037 
22. González E, Molina T, Luttges C. Características de la educación sexual escolar recibida y su asociación con la edad de inicio sexual y uso de anticonceptivos en adolescentes chilenas sexualmente activas. Rev Chil. Obstet Ginecol. 2015;80:24-32.

23. Chiavegatto FAD, Kawachi I. Income inequality is associated with adolescent fertility in Brazil: a longitudinal multilevel analysis of 5565 municipalities. BMC Public Health. 2015;15:103. Doi: 10.1186/ s12889-015-1369-2.

24. Atienzo EE, Campero L, Lozada AL, Herrera C. Aspiraciones educativas y familiares como condicionantes en la prevención de embarazos tempranos en México. Salud Publica Mex. 2014;56:286-94.
25. Salvador J, Madariegue E. Gestación en adolescentes: experiencia en el Hospital nacional Cayetano Heredia 1992-1994. Ginecol Obstet. 1995;41:37-47.

26. Huanco D, Ticona M, Ticona M, Huanco F. Frecuencia y repercusiones maternas y perinatales del embarazo en adolescentes atendidas en hospitales del Ministerio de Salud del Perú, año 2008. Rev Chil Obstet Ginecol. 2012;77:122-8.

27. Menéndez GE, Navas I, Hidalgo Y, Espert J. El embarazo y sus complicaciones en la madre adolescente. Revista Cubana de Obstetricia y Ginecología. 2012;38:333-42. 